\title{
Transtornos psiquiátricos na gestação e no puerpério: classificação, diagnóstico e tratamento
}

\section{Psychiatry disorders in pregnancy and puerperium: classification, diagnosis and treatment}

\author{
Renata Sciorilli CAMACHO ${ }^{1}$ \\ Fábio Scaramboni Cantinelli ${ }^{1}$ \\ CARMEN SyLVIa RibEIRO ${ }^{1}$ \\ AMAURY CANTILINO ${ }^{2}$ \\ Bárbara Karina Gonsales ${ }^{3}$ \\ ÉRIKA BRAGUITTONI ${ }^{3}$ \\ Joel RenNó JR. ${ }^{4}$
}

\begin{abstract}
Resumo
Diversas questões ainda estão em aberto no que se refere a um tema tão amplo quanto a saúde mental das mulheres em período de gestação e puerpério. Por mais contraditório que possa parecer, muitas pacientes apresentam tristeza ou ansiedade em vez de alegria nessas fases de suas vidas. Os limites entre o fisiológico e o patológico podem ser estreitos, o que pode gerar dúvidas em obstetras, clínicos ou psiquiatras. Muitas pacientes também sentem-se culpadas, prejudicando a aderência ao tratamento e a aceitação de uma patologia em uma fase que, em tese, deveria ser de alegria.

Nas últimas décadas, estudos têm investigado um pouco mais sobre o tema, mas algumas questões ainda estão em debate: os transtornos puerperais poderiam ser uma manifestação de um transtorno prévio não adequadamente tratado? Seriam a gestação ou o puerpério fatores protetores ou de risco para o desencadeamento de transtornos psiquiátricos? As alterações hormonais que ocorrem nesse período poderiam estar envolvidas na sua etiologia? Quais seriam os principais fatores de risco? Em quais situações seria adequado usar psicofármacos como medida de tratamento?

Neste artigo, serão abordadas algumas dessas questões, sobre um tema que ainda precisa ser muito investigado para que tenhamos conclusões mais precisas.
\end{abstract}

Palavras-chave: Gestação, puerpério, transtornos psiquiátricos, fatores de risco, tratamento

\section{Abstract}

Several questions regarding mental health during the period of pregnancy and puerperium are still open. Even this seems contradictory, many patient present sadness or anxiety instead

Recebido: 20/03/2006 - Aceito: 27/03/2006

1 Médico psiquiatra. Colaborador do Projeto de Atenção à Saúde Mental da Mulher (Pró-Mulher) do Instituto de Psiquiatria do Hospital das Clínicas da Faculdade de Medicina da Universidade de São Paulo (IPq-HC-FMUSP).

2 Médico psiquiatra. Coordenador do Programa de Depressão na Mulher do Hospital das Clínicas da Universidade Federal de Pernambuco (HC-UFPE).

3 Psicóloga clínica. Colaboradora do Pró-Mulher do IPq-HC-FMUSP.

4 Médico psiquiatra. Coordenador do Pró-Mulher do do IPq-HC-FMUSP.

Endereço para correspondência: Renata Sciorilli Camacho. Rua Atlântica, 400, Jardim do Mar - 09750480 - São Bernardo do Campo (SP). Fone: (11) 4330-4487. E-mail: sc_camacho@hotmail.com 
of joy in these phases of life. The limits between physiological and the pathological one can be narrow, what it may generate doubts in obstetricians, physicians or psychiatrists. Many patients also feel guilty, harming the treatment and the acceptance of her pathology in a phase that theoretically would have to be of joy.

Few decades ago till today, there are studies that investigate deeper on this topic, but some questions still are in discussion: the puerperal diseases could be a manifestation of a previous disease not adequately treated? Would be the pregnancy or the puerperium protective factors or risk factors for the psychiatric diseases development? Could be involved in the etiology the hormonal alterations that occur in this period? Which would be the main factors of risk? In which situations it would be adjusted to use medications as treatment?

This article has the proposal to discuss some of these questions that are immersed on a topic that is still opening to investigation to let us with more accuracy conclusions.

Key-words: Psychiatric pregnancy, puerperium, diseases, risk factors, treatment.

\section{Introdução}

A gestação e o puerpério são períodos da vida da mulher que precisam ser avaliados com especial atenção, pois envolvem inúmeras alterações físicas, hormonais, psíquicas e de inserção social, que podem refletir diretamente na saúde mental dessas pacientes.

Tem-se dado importância cada vez maior ao tema, e estudos atuais têm visado delinear os fatores de risco para os transtornos psiquiátricos nessas fases da vida, a fim de se realizarem diagnóstico e tratamento o mais precocemente possível.

Estudos recentes revelaram que transtornos psiquiátricos subdiagnosticados e não tratados em gestantes podem levar a graves conseqüências maternofetais, até mesmo durante o trabalho de parto (Jablensky et al., 2005; Seng et al., 2001).

Sabe-se ainda que a presença de ansiedade ou depressão na gestação está associada a sintomas depressivos no puerpério (Bloch et al., 2003).

Esses temas têm sido mais pesquisados nas últimas décadas e ainda não há especificação para essas patologias na Classificação Internacional de Doenças (CID-10), a não ser como diagnóstico de exclusão, no caso dos transtornos relacionados ao puerpério.

O Manual diagnóstico e estatístico de transtornos mentais, na sua quarta edição e com texto revisado (DSM-IV-TR), não distingue os transtornos do humor do pós-parto dos que acontecem em outros períodos, exceto como especificador “com início no pós-parto”, que é utilizado quando o início dos sintomas ocorre no período de quatro semanas após o parto (Cantilino, 2003).

Este artigo tem como objetivo uma revisão da literatura, apresentando os principais aspectos epidemiológicos, de classificação, diagnóstico e tratamento dos transtornos psiquiátricos relacionados à gestação e ao puerpério.

\section{Epidemiologia}

Apesar de a gestação ser tipicamente considerada um período de bem-estar emocional e de se esperar que a chegada da maternidade seja um momento jubiloso na vida da mulher, o período perinatal não a protege dos transtornos do humor.

Estima-se uma prevalência de depressão na gravidez da ordem de $7,4 \%$ no primeiro, $12,8 \%$ no segundo e $12 \%$ no terceiro trimestre (Bennett et al., 2004).

Nas adolescentes, foi verificada prevalência entre $16 \%$ e $44 \%$, quase duas vezes mais elevada que nas gestantes adultas, o que pode estar relacionado à falta de maturidade afetiva e de relacionamentos dessas pacientes, bem como ao fato de grande parte delas terem que abandonar seus estudos em razão da maternidade (Szigethy e Ruiz, 2001).

A disforia no pós-parto (maternity blues) inclui sintomas depressivos leves e pode ser identificada em $50 \%$ a $85 \%$ das puérperas, dependendo dos critérios diagnósticos utilizados (Cantilino, 2003).

Um estudo com 1.558 mulheres detectou $17 \%$ das gestantes com sintomas significativos para depressão na gestação tardia, $18 \%$ no puerpério imediato e $13 \%$ entre a sexta e a oitava semanas do puerpério. $\mathrm{O}$ mesmo valor (13\%) foi encontrado no sexto mês do puerpério (Josefsson et al., 2001).

Um outro estudo constatou valores oscilando entre $8,6 \%$ e $10,1 \%$ para o diagnóstico de depressão entre a $6^{a}$ e a 24를 semanas do puerpério (Boyce e Hickey, 2005).

Uma metanálise de 59 estudos mostrou uma estimativa de prevalência de depressão pós-parto da ordem de 13\% (O’Hara e Swain, 1996).

A psicose puerperal é um quadro mais raro, e a incidência encontrada foi entre 1,1 e 4 para cada 1.000 nascimentos (Bloch et al., 2003).

\section{Fatores de risco}

Os principais fatores de risco psicossociais relacionados à depressão maior no puerpério são: idade inferior a 16 anos, história de transtorno psiquiátrico prévio, eventos estressantes experimentados nos últimos 12 meses, conflitos conjugais, ser solteira ou divorciada, estar desempregada (a paciente ou o seu cônjuge) e apresentar pouco suporte social.

Outros fatores de risco apontados foram: personalidade vulnerável (mulheres pouco responsáveis ou 
organizadas), esperar um bebê do sexo oposto ao desejado, apresentar poucas relações afetivas satisfatórias e suporte emocional deficiente (Boyce e Hickey, 2005). Abortamentos espontâneos ou de repetição também foram indicados como fatores de risco (Botega, 2006).

Mulheres mais suscetíveis aos transtornos do humor no puerpério também teriam diagnóstico de transtorno disfórico pré-menstrual ou apresentaram sintomas depressivos no segundo ou quarto dia do pósparto. Pacientes que tiverem história de sensibilidade aumentada ao uso de anticoncepcionais orais também seriam mais vulneráveis (Bloch et al., 2005).

Um outro estudo tentou encontrar uma relação entre a gravidez indesejada e o risco para depressão, mas os resultados foram pouco conclusivos. Verificou-se apenas que mulheres com escolaridade mais alta e melhor rendimento financeiro apresentavam menor risco, enquanto mulheres provenientes de famílias populosas apresentavam elevado risco para depressão. Levantouse também a hipótese de que a existência prévia de transtorno mental pode ser o mais importante fator associado ao risco de se apresentarem novos episódios no puerpério (Schmiege e Russo, 2005).

Mulheres portadoras de transtorno afetivo bipolar apresentaram risco elevado de psicose puerperal (260/1.000) quando comparadas a mulheres saudáveis, que apresentavam 1 a 2 casos a cada 1.000 puérperas (Chaudron e Pies, 2003).

\section{Etiologia}

A etiologia da depressão puerperal ainda não é completamente conhecida, mas acredita-se que, além dos fatores de risco anteriormente mencionados, fatores hormonais e hereditários também estejam envolvidos.

Na gestação, os níveis de estrógeno e progesterona são superiores àqueles vistos nas mulheres fora do período gestacional e esse fator pode estar envolvido nas alterações do humor que ocorrem nessa fase. A queda brusca desses hormônios no pós-parto estaria envolvida na etiologia da depressão puerperal. Bloch $e t$ al. (2003) realizaram uma revisão da literatura a fim de correlacionarem os fatores endócrinos e hereditários com a etiologia desse transtorno. Não se constatou relação genética diferente daquela já esperada em quadros não puerperais. Levantou-se a hipótese de que algumas mulheres seriam mais sensiveis a variações hormonais em qualquer momento de suas vidas, incluindo-se período pré-menstrual, menarca, gestação, puerpério, menopausa e até mesmo durante o uso de anticoncepcionais.

\section{Transtorno do pânico}

Um estudo comparou a sintomatologia em mulheres com transtorno do pânico diagnosticado previamente e constatou que $43 \%$ dessas pacientes apresentaram
Tabela 1. Principais fatores de risco para 0 desenvolvimento de depressão na gestação.

\begin{tabular}{l|l}
\hline Biológicos & Psicossociais \\
\hline $\begin{array}{l}\text { História de transtorno do } \\
\text { humor ou ansiedade }\end{array}$ & Abuso sexual na infância \\
\hline $\begin{array}{l}\text { História de depressão } \\
\text { pós-parto }\end{array}$ & Gravidez precoce \\
\hline $\begin{array}{l}\text { História de transtorno } \\
\text { disfórico pré-menstrual }\end{array}$ & Gravidez não planejada \\
\hline $\begin{array}{l}\text { Doença psiquiátrica na } \\
\text { família }\end{array}$ & $\begin{array}{l}\text { Gravidez não desejada ou } \\
\text { não aceita }\end{array}$ \\
\hline & Mães solteiras \\
\hline & Ter muitos filhos \\
\hline & Reduzido suporte social \\
\hline & $\begin{array}{l}\text { Violência doméstica ou } \\
\text { conflitos no lar }\end{array}$ \\
\hline & Baixo nível de escolaridade \\
\hline
\end{tabular}

Adaptado de Ryan et al., 2005.

melhora dos sintomas ansiosos na gestação, 33\%, piora e 24\% não apresentaram nenhuma alteração. Já no sexto mês do puerpério, $63 \%$ tiveram piora dos sintomas de pânico (Northcott e Stein, 1994).

Um outro estudo constatou que o puerpério seria um período de maior vulnerabilidade para o desencadeamento do primeiro episódio de pânico. Entre as mulheres estudadas, 10,9\% apresentaram início dos sintomas no puerpério (neste caso, até 12 semanas do puerpério), um número maior do que o esperado, que é de 0,92\% (Sholomskas et al., 1993).

\section{Transtorno obsessivo-compulsivo (TOC)}

Das gestantes portadoras de TOC, $46 \%$ apresentaram piora da sintomatologia na primeira gestação e $50 \%$, na segunda gestação. Sintomas de TOC são freqüentes no pós-parto e incluem pensamentos e obsessões relacionados a possíveis contaminações da criança e pensamentos obsessivos negativos em relação ao trabalho de parto (Labad et al., 2005).

A gestação também está associada ao aparecimento do TOC em $13 \%$ das mulheres e constatou-se piora da sintomatologia em 29\% das puérperas. Mulheres portadoras de TOC também apresentam elevado risco para o desenvolvimento de depressão pós-parto (Williams e Koran, 1997).

\section{Transtornos puerperais}

Os transtornos psiquiátricos puerperais são caracteristicamente classificados como: disforia do pós-parto 
(puerperal blues), depressão pós-parto e psicose puerperal (Chaudron e Pies, 2003).

A puerperal blues costuma acometer as mulheres nos primeiros dias após o nascimento do bebê, atingindo um pico no quarto ou quinto dia após o parto e remitindo de maneira espontânea, no máximo, em duas semanas. Inclui choro fácil, labilidade do humor, irritabilidade e comportamento hostil para com familiares e acompanhantes. Esses quadros normalmente não necessitam de intervenção farmacológica, e a abordagem é feita no sentido de manter suporte emocional, compreensão e auxílio nos cuidados com o bebê (Cantilino, 2003). Evidências sugerem que a depressão pós-parto pode ser parte ou continuação da depressão iniciada na gestação (Ryan et al., 2005).

Os sinais e sintomas de depressão perinatal são pouco diferentes daqueles característicos do transtorno depressivo maior não psicótico que se desenvolvem em mulheres em outras épocas da vida. As pacientes apresentam-se com humor deprimido, choro fácil, labilidade afetiva, irritabilidade, perda de interesse pelas atividades habituais, sentimentos de culpa e capacidade de concentração prejudicada. Sintomas neurovegetativos, incluindo insônia e perda do apetite, são descritos com frequiência (Nonacs e Cohen, 1998; Gold, 2002). Contudo, alguns sintomas somáticos podem ser confundidos com situações normais desse período. Assim, sintomas como hipersonia, aumento de apetite, fadigabilidade fácil, diminuição do desejo sexual e queixas de dor e desconfortos em diferentes sistemas são de pouca utilidade para o diagnóstico de depressão nessa fase.

É importante frisar que muitas mulheres com depressão perinatal não revelam seus sintomas de depressão com receio de possível estigmatização (Epperson, 1999). As mulheres sentem que as expectativas sociais são de que elas estejam satisfeitas e acabam sentindo-se culpadas por estarem experimentando sintomas depressivos num momento que deveria ser de alegria.

Algumas particularidades clínicas são descritas na depressão pós-parto. Há sugestão de haver um componente ansioso mais proeminente (Ross et al., 2003), além de pensamentos recorrentes de causar danos ao bebê (Jennings et al., 1999). Wisner et al. (1999) analisaram pensamentos obsessivos em mulheres deprimidas e observaram que mulheres com depressão pós-parto tinham mais pensamentos de agressividade contra seus filhos do que mulheres deprimidas fora do período pós-parto, independentemente da gravidade do quadro. Godfroid et al. (1997), em estudo polissonográfico em mulheres com depressão, mostraram que mulheres com depressão pós-parto tinham aumento significativo na fase IV do sono e menor tempo de estágio I quando comparadas a mulheres com depressão de gravidade semelhante fora do período pós-parto. Hendrick et al. (2000), também numa comparação entre mulheres com depressão no pós-parto e fora dele, observaram que a depressão pós-parto requer mais tempo para responder à farmacoterapia e tende a necessitar de mais agentes antidepressivos para se obter remissão dos sintomas. Além disso, é frequiente o relato de sentimentos ambivalentes acerca do bebê e de opressão pela responsabilidade de cuidar dos filhos (Beck, 1996; Nonacs e Cohen, 2000). Esses dados apresentam-se em favor da especificidade do conceito de depressão pós-parto.

A psicose puerperal costuma ter início mais abrupto. Estudos verificaram que 2/3 das mulheres que apresentaram psicose puerperal iniciaram sintomatologia nas duas primeiras semanas após o nascimento de seus filhos. Descreve-se um quadro com presença de delírios, alucinações e estado confusional que parece ser peculiar aos quadros de psicose puerperal. Pode haver sintomas depressivos, maníacos ou mistos associados. Não foi estabelecida nenhuma apresentação típica. No entanto, essas mulheres costumam apresentar comportamento desorganizado e delírios que envolvem seus filhos, com pensamentos de lhes provocar algum tipo de dano. Apesar de o suicídio ser raro no período puerperal em geral, a incidência deste nas pacientes com transtornos psicóticos nesse período é alta, necessitando muitas vezes de intervenção hospitalar por esse motivo, bem como pelo risco de infanticídio. Sintomas depressivos, mais do que maníacos, em geral estão associados aos quadros em que ocorrem infanticídio ou suicídio (Chaudron e Pies, 2003).

Estudos neurocientíficos recentes sustentam a hipótese de que a mulher portadora de psicose puerperal que comete infanticídio necessita mais de tratamento e reabilitação do que de punição legal, a fim de se evitarem outras fatalidades decorrentes da gravidade do quadro; atualmente, alguns países já defendem essa hipótese. A educação familiar também estaria presente nesse tipo de intervenção (Spinelli, 2004).

\section{Sobre escalas de depressão para uso na gravidez}

Até onde sabemos, não existem escalas desenhadas especificamente para a detecção de depressão durante a gravidez. Autores têm utilizado a Edinburgh Postnatal Depression Scale (EPDS) com essa finalidade (Clark, 2000; Areias et al., 1996). Um estudo americano testou o Inventário de Depressão de Beck (BDI) - uma escala para depressão geral - durante a gravidez e obteve o seguinte resultado com o ponto de corte em 16: sensibilidade de $83 \%$, especificidade de $89 \%$, valor preditivo positivo de $50 \%$ e valor preditivo negativo de $98 \%$ (Holcomb et al., 1996). Provavelmente, o autor obteve um baixo valor preditivo positivo pelo fato de o BDI conter itens de sintomas físicos que se confundem com um quadro normal da gravidez.

Também têm sido utilizadas na gravidez escalas que abordam preditores de depressão pós-parto. $\mathrm{O}$ Pregnancy Risk Questionnaire é uma escala de 18 itens que lista fatores de risco psicossociais para depressão pós-parto. Em estudo de validação original, obteve 
sensibilidade de $44 \%$ e valor preditivo positivo de $23 \%$ (Austin et al., 2005). Esses valores parecem bem razoáveis para um instrumento de predição. Um outro instrumento com a mesma finalidade é o Postpartum Depression Predictors Inventory, uma checklist que idealmente deve ser preenchida em cada um dos três trimestres de gravidez e que também acessa fatores de risco psicossociais (Beck, 1998).

\section{Detecção das mulheres com depressão pós-parto}

Mulheres no puerpério com frequiência são examinadas por seus obstetras ou clínicos gerais em consultas focadas na recuperação física após o parto. Além disso, são vistas por pediatras dos seus filhos de quatro a seis vezes durante $o$ ano seguinte ao nascimento de seu bebê. Quando apresentam depressão, embora busquem ajuda mais comumente com esses médicos do que com profissionais de saúde mental (Gold, 2002), muitas vezes não são diagnosticadas ou reconhecidas como deprimidas de forma adequada. Trabalhos recentes vêm mostrando a utilidade do uso de escalas de auto-avaliação para triagem de mulheres com depressão pós-parto em serviços de atendimento primário (Buist et al., 2002). A possibilidade de detecção de depressão pós-parto com essas escalas tem-se mostrado significativamente maior que a detecção espontânea durante avaliações clínicas de rotina por médicos nesses serviços (Fergerson et al., 2002). As escalas serviriam para alertar clínicos, obstetras e pediatras para aquelas mulheres que possivelmente precisariam de avaliação mais profunda e tratamento.

No Brasil, onde os médicos cada vez mais se vêem obrigados a fazer atendimentos de um grande número de pacientes com uma disponibilidade de tempo pequena, instrumentos desse tipo acabam tendo valia considerável. Ademais, essas escalas são auto-aplicáveis e de fácil utilização por profissionais não médicos e sem especialização em saúde mental. Essas características fazem que o procedimento de aplicação destas seja, além de prático, considerado de baixo custo, o que torna viável a sua utilização em serviços de atenção primária à saúde.

Pensando nessa praticidade, foram criadas duas escalas desenhadas especificamente para rastreamento de depressão pós-parto: a EPDS, em 1987, e a Postpartum Depression Screening Scale (PDSS), em 2000. Ambas já possuem tradução para o português e validação no Brasil, sendo o estudo da PDSS realizado por um dos autores deste artigo (Cantilino, 2003).

A EPDS (Cox et al., 1987) foi o primeiro instrumento encontrado na literatura desenvolvido para triar especificamente a depressão pós-parto. A EPDS é um instrumento de auto-registro que contém 10 questões de sintomas comuns de depressão e que utiliza formato de respostas do tipo Likert. A mãe escolhe as respostas que melhor descrevem o modo como tem se sentido na última semana.
Realizando um estudo fenomenológico de depressão pós-parto, Beck (1992) percebeu temas que descreviam mais sobre a essência dessa experiência. Esses temas tinham relação com solidão insuportável, pensamentos obsessivos, sensação de estar fora de si, culpa sufocante, dificuldades cognitivas, perda de interesses prévios, ansiedade incontrolável, insegurança, perda de controle das emoções e idéias relacionadas à morte. Um outro estudo fenomenológico conduzido por Beck (1996) investigou especificamente o significado de experiências de mães com depressão pós-parto que tinham interagido com seus bebês e crianças mais velhas. Num estudo de avaliação de instrumentos para depressão em mulheres no pós-parto, Ugariza (2000) assinalou a necessidade de uma escala de avaliação que pudesse mensurar sintomas específicos de depressão pós-parto. Considerando as observações feitas nos estudos e procurando maior acurácia na triagem de pacientes com depressão pós-parto, idealizou-se a criação de uma nova escala que abrangesse esses conceitos.

A PDSS, desenvolvida por Beck e Gable (2000), nos Estados Unidos, é uma escala de auto-avaliação do tipo Likert. $\mathrm{O}$ instrumento tem 35 itens que avaliam sete dimensões: distúrbios do sono/apetite, ansiedade/ insegurança, labilidade emocional, prejuízo cognitivo, perda do eu, culpa/vergonha e intenção de causar dano a si. Cada dimensão é composta de cinco itens que descrevem como uma mãe pode estar se sentindo após o nascimento de seu bebê (Beck e Gable, 2000).

Um estudo foi conduzido fazendo-se uma análise comparativa da performance da PDSS com a EPDS (Beck e Gable, 2001). Um total de 150 mães no pós-parto preencheu os dois instrumentos e, logo em seguida, foi submetido à entrevista para diagnóstico no DSM-IV. Dezoito dessas mulheres (12\%) foram diagnosticadas como tendo depressão maior, 28 (19\%) tinham depressão menor e 104 (69\%) não tinham depressão. Utilizando os escores de pontos de corte para depressão maior recomendados nas publicações dos dois instrumentos, a PDSS conseguiu a melhor combinação de sensibilidade, $94 \%$, e especificidade, $91 \%$, entre as três escalas. A PDSS identificou 17 das mulheres (94\%) diagnosticadas com depressão pós-parto e a EPDS, 14 dessas mulheres (78\%). O diferencial da sensibilidade observado pode se dever ao fato de a PDSS abordar outros componentes desse transtorno do humor.

No Brasil, a EPDS foi traduzida para o português e validada por Santos et al. (2000). Sessenta e nove puérperas foram selecionadas de uma amostra maior para preencherem o instrumento e serem entrevistadas. Usando o ponto de corte recomendado de $11 / 12$, obtevese sensibilidade de $72 \%$, especificidade de $88 \%$ e valor preditivo positivo de $78 \%$. O instrumento foi validado em Brasília - que, segundo o Programa das Nações Unidas para o Desenvolvimento (2003), é uma cidade que apresenta um dos maiores índices de desenvolvimento humano do Brasil - e a maior parte da amostra 
(72\%) foi composta por mulheres com renda familiar média-alta ou alta. Como os baixos índices de desenvolvimento de algumas regiões brasileiras implicam baixa escolaridade, um fato também comum na periferia das grandes metrópoles, a própria autora do estudo sugere a validação da EPDS em outras regiões e com amostras de classes média e média-baixa para completar a análise do instrumento no Brasil.

Assim, foi realizado estudo de validação da EPDS, em Recife, com 120 puérperas, 60 delas de um serviço público e 60 de um serviço privado. A sensibilidade foi de $94 \%$, a especificidade, de $85 \%$, o valor preditivo negativo, de $99 \%$, mas o valor preditivo positivo foi baixo, $48 \%$ (Cantilino et al., 2003).

A PDSS também teve uma tradução sistematizada e foi submetida a estudo de validação no Brasil. Nesse estudo, 120 puérperas foram avaliadas (60 num serviço público e 60 num serviço privado). A PDSS apresentou sensibilidade de $94 \%$, especificidade de $95 \%$, valor preditivo positivo de $75 \%$ e valor preditivo negativo de $99 \%$. Os resultados presentes confirmam sua validade da PDSS na população brasileira para rastreamento de depressão pós-parto (Cantilino, 2003; Cantilino et al., no prelo).

\section{Importância do diagnóstico e tratamento precoces}

O infanticídio e o suicídio estão entre as complicações mais graves decorrentes de transtornos puerperais sem intervenção adequada. No entanto, a existência de transtornos psiquiátricos não só no puerpério, mas também na gestação, pode levar a outras graves consequiências.

Mulheres com diagnóstico de esquizofrenia ou depressão maior apresentaram elevado risco para complicações na gravidez, trabalho de parto e período neonatal. Entre essas complicações, há anormalidades placentárias, hemorragias e sofrimento fetal. Mulheres com esquizofrenia apresentam risco elevado para descolamento prematuro de placenta e, mais frequientemente, tiveram filhos com baixo peso ao nascer. Essas crianças também apresentaram malformações cardiovasculares e menor circunferência encefálica do que os filhos de mães saudáveis (Jablensky et al., 2005).

Seng et al. (2001) constataram que mulheres portadoras de transtorno de estresse pós-traumático apresentavam risco elevado para gravidez ectópica, abortamento espontâneo, hiperemese gravídica, contrações uterinas prematuras e crescimento fetal excessivo.

O relacionamento mãe-filho também demonstrou estar prejudicado quando foram avaliadas 507 mães e seus filhos, aos 3 meses de idade. Os filhos de mães que apresentaram diagnóstico de depressão pós-parto tinham dificuldades para dormir e se alimentar. Apresentavam também prejuízos de interação corporal com o ambiente e sorriso social diminuído. Essas pacientes queixavam-se com muita freqüência de cansaço excessivo, o que acabava refletindo de forma negativa no relacionamento com seus filhos e, por conseqüencia, no desenvolvimento deles (Riguetti-Veltema et al., 2002).

Deve-se, portanto, avaliar cada caso com especial atenção, a fim de estabelecer-se a melhor estratégia de tratamento para cada situação em particular, da maneira mais precoce possível.

\section{Opções de tratamento}

Pesquisas têm utilizado técnicas de tratamento psicofarmacológico, psicossocial, psicoterápico e tratamentos hormonais, além da eletroconvulsoterapia (ECT), indicada para casos mais graves ou refratários a outras formas de tratamento. A maioria das intervenções psicossociais e hormonais tem-se mostrado pouco eficiente. Entretanto, resultados de estudos focados sobre psicoterapia interpessoal, estratégias cognitivocomportamentais e intervenções farmacológicas têm-se evidenciado eficientes.

\section{Uso de psicofármacos durante gestação e lactação}

A decisão de oferecer tratamentos biológicos às gestantes é um processo decisório complexo que envolve uma interação constante entre paciente, família, obstetra e psiquiatra. Estabelecer uma aliança terapêutica é fundamental. A confiança que a gestante deposita em seus médicos certamente minimizarará qualquer percalço, principalmente os efeitos colaterais que podem ocorrer durante o tratamento. Essa decisão sempre deve levar em conta, como sua mola mestra, a relação risco-benefício.

Wisner et al. (2000) publicaram uma importante revisão ressaltando o tema. Entre os vários conceitos levantados por essa equipe estavam a essencialidade do consentimento informado e o respeito pelos valores e pelas preferências da paciente por parte do médico, que sempre colocará com clareza sua experiência na discussão, ou seja, o médico oferece suporte e soluções aos problemas levantados, já que a paciente é convocada a participar da melhor forma possível da decisão, que, em geral, deixa inteiramente a cargo do médico.

Diante de uma história clínica a mais completa possível, as opções de tratamento são oferecidas, incluindose a de não tratar. Entre as opções estão os tratamentos biológicos (os medicamentos são descritos levando-se em consideração a sua eficácia e os riscos particulares para mãe e feto). Os possíveis riscos envolvem toxicidade fetal, considerando-se a morte intra-uterina, malformações físicas, prejuízo de crescimento, teratogenicidade comportamental e toxicidade neonatal.

Algo que deve ser mencionado com veemência à gestante é que o fato de não tratar poderá trazer muito mais danos ao feto, pelo que haverá, devido ao estresse, efeito sobre o eixo hipotálamo-hipófise-adrenal, com aumento de cortisóides e danos ao produto conceptual. 
Situações extremas, como tentativa ou risco elevado de suicídio ou um estado psicótico no qual a paciente não responde, até mesmo do ponto de vista legal, pelos seus atos, tenderão à decisão de medicação compulsória.

Como o risco de teratogenicidade é a principal preocupação da gestante, a Food and Drug Administration (FDA), órgão norte-americano que controla alimentos e fármacos, estabeleceu uma classificação, segundo esse risco, para cada psicofármaco:

- Risco A: estudos controlados não demonstram risco. Estudos adequados e bem controlados em gestantes não têm demonstrado ou evidenciado nenhum risco ao feto.

- Risco B: sem evidência de risco em humanos. Ou os achados em animais demonstram risco, mas os achados em humanos não, ou se estudos adequados em humanos não têm sido realizados, achados em animais são negativos.

- Risco C: risco não pode ser excluído. Faltam estudos em humanos, e os estudos em animais são positivos para o risco fetal ou estão ausentes também. Contudo, potenciais benefícios podem justificar o risco potencial.

- Risco D: evidência positiva de risco. Dados de investigação ou relatados, posteriormente, mostram risco ao feto. Ainda assim, potenciais benefícios podem ter mais valor que o risco em potencial.

- Risco X: contra-indicação absoluta em gravidez. Estudos em animais ou humanos de investigação, ou relatados posteriormente, mostram um risco fetal que claramente suplanta qualquer possível benefício à paciente.

Dessa maneira, é fácil concluir que o tempo que uma determinada medicação possui no mercado, ou seja, a experiência a ela associada, é fundamental nessa classificação, e a segurança oferecida pelas medicações mais novas muitas vezes ainda não pode ser traduzida em relação ao seu uso em gravidez.

\section{Particularidades de cada classe de psicofármacos}

Descrever o risco inerente a cada droga de cada grupo psicofarmacêutico seria longo e fugiria em parte do contexto deste artigo. Concentra-se atenção nas mais conhecidas e usadas. Os dados usados a seguir são do Commitee on Drugs of American Academy of Pediatrics (2000) e de Bazire (2002).

\section{Antidepressivos}

Os antidepressivos tricíclicos estão há muito tempo em uso e, portanto, a sua segurança tem sido estabelecida ao longo dos anos, particularmente a amitriptilina (risco B). A questão pode ficar por conta dos efeitos hipotensivos sobre a mãe, o que indicaria preferência pela desipramina (risco $\mathrm{C}$ ), já que a nortriptilina tem risco D. Os inibidores seletivos da recaptura da serotonina (IRSS), em geral, também têm tido sua segurança bem estabelecida ao longo do tempo, embora tenham, obviamente, menos tempo de mercado que os tricíclicos. A “irmã mais velha”, fluoxetina, é largamente usada e tem risco $\mathrm{B}$; alguma restrição ficaria por conta de sua meia-vida bastante longa, ou seja, numa eventual necessidade de retirada, a droga ainda permaneceria algum tempo no organismo. A paroxetina, que até recentemente era bastante utilizada em gestantes, teve a sua segurança contestada em artigo de Williams e Wooltorton (2005), que apontaram risco teratogênico bastante importante para essa droga, que está sendo reclassificada como risco $\mathrm{D}$, devendo ser, portanto, utilizada em último caso. Particularmente seguros e cada vez mais utilizados são a sertralina e o citalopram, com o estabelecimento de sua segurança cada vez maior (risco B). A fluvoxamina tem risco C, assim como o escitalopram, embora não haja relato de teratogenicidade para essa droga.

Os inibidores da monoaminooxidase (IMAOs) também têm risco $\mathrm{B}$ atribuído, seus efeitos hipotensores, em particular da tranilcipromina, além da própria questão dietética, seriam restritivos. Venlafaxina, mirtazapina e reboxetina têm classificação $\mathrm{C}$, e seu uso em gestação também tem aumentado. Duloxetina é uma droga muito recente e não encontraria uma classificação ainda apropriada. A opção antidepressiva de risco A seria o uso do triptofano, de eficácia discutível.

\section{Estabilizadores do humor e anticonvulsivantes}

O carbonato de lítio (risco D) é bem associado a malformações, em particular cardiovasculares - especialmente a anomalia de Ebstein -, e o seu uso é proibido no primeiro trimestre. Pode ser utilizado nos segundo e terceiro trimestres com muita indicação, esgotadas outras possibilidades. Carbamazepina e oxcarbazepina têm risco $\mathrm{C}$ associado, mas parecem ser a melhor opção, particularmente para o primeiro trimestre, já que 0 valproato/divalproato atravessa facilmente a placenta $\mathrm{e}$ tem sido ligado a algumas malformações, em particular a espinha bífida (risco D). Fenitoína também tem risco $\mathrm{D}$, pois também atravessa a membrana fetal e é associada a síndrome fetal hidantoínica, caracterizada por retardo do crescimento, retardo mental, defeitos faciais, hirsurtismo, anomalias cardiovasculares, geniturinárias e gastrointestinais. Fenobarbital tem clara implicação teratogênica (risco D). Lamotrigina e vigabatrina têm relativamente pouco tempo de uso, e a classificação é C. Topiramato também é $\mathrm{C}$, embora demonstre alguma teratogenicidade em ratos.

\section{Antipsicóticos}

Os antipsicóticos de gerações mais recentes têm, em geral, a preferência no tratamento, em especial por sua 
eficácia em sintomas negativos. Destes, a risperidona usufrui de mais tempo no mercado, e riscos não the têm sido atribuídos, embora ainda apresente risco C. Quetiapina e olanzapina também têm alguma segurança associada (risco C), pois não se fez ainda nenhuma associação de teratogenicidade. A quetiapina é a menos estudada até o momento, mas pode ser interessante por sua falta de ação sobre a prolactina, assim como a clozapina (risco B), particularmente segura, mas com a limitação da farmacovigilância relacionada à agranulocitose, além de efeitos hipotensores e grande sedação. O haloperidol e as fenotiazinas (levomepromazina, clorpromazina) apresentam segurança relativamente bem estabelecida, pelo tempo de mercado que possuem (risco B). O haloperidol chegou a ser usado, em doses baixas, como alternativa para hiperemese gravídica. Possíveis limitações podem residir nos efeitos colaterais.

\section{Benzodiazepínicos}

Têm tido alguma teratogenicidade associada, embora os dados sejam controversos pela sua freqüente associação com álcool e drogas ilícitas. Alprazolam não traz relatos formais de teratogenicidade (risco C). Clordiazepóxido (risco D) atravessa a placenta e tem sido relacionado à teratogenicidade, em particular se usado nos primeiros 42 dias da gestação. Diazepam e lorazepam são associados de forma costumeira, quando usados no último trimestre, a recém-nascidos hipotônicos ou com lentificação de respostas, incluindo o reflexo para mamar; por isso, seriam classificados como risco D. Clonazepam apresenta relativa segurança e recebe a classificação C. Dos demais benzodiazepínicos, não se têm muitas informações estabelecidas.

\section{Uso de psicofármacos durante a lactação}

Atualmente, muitos antidepressivos estão sendo estudados em relação à lactação, e os ISRS foram os menos presentes no leite materno. Entre eles, a sertralina e a paroxetina parecem ser as melhores alternativas (Haberg e Matheson, 1997). Também foram consideradas seguras (de baixo risco) as seguintes drogas: sulpirida, a maioria dos tricíclicos, triptofano, moclobemida, benzodiazepínicos em dose baixa e única, zolpidem, carbamazepina, valproato em doses baixas e fenitoína.

Drogas como haloperidol, fenotiazinas em baixas doses, IMAOS, mirtazapina, ISRS, trazodona, benzodiazepínicos e betabloqueadores foram consideradas de risco moderado.

A clozapina é contra-indicada pelo risco de agranulocitose, e estudos realizados em animais detectaram-na no leite. Antipsicóticos de última geração ainda têm poucos estudos, devendo-se então não utilizá-los. Deve-se evitar também o uso do carbonato de lítio pelo risco de toxicidade no bebê. Ainda há poucos estudos relacionados aos novos antidepressivos para que possam ser utilizados com segurança (Botega et al., 2006).

\section{Tratamento hormonal}

O tratamento hormonal nos transtornos do humor é muito antigo, e atualmente o uso de estrógenos sublinguais e transdérmicos, como o 17-B estradiol tem sido testado no tratamento da depressão pós-parto (Murray, 1996). Embora esses relatos proponham que o uso de estrógenos tenha bons resultados, apenas números modestos de melhora sintomática foram apresentados. Acredita-se que os estrógenos atuem pela interação com receptores do núcleo e de membranas celulares (McEwen, 1999). A ação desses hormônios sobre receptores de membrana representaria um papel importante na síntese, na liberação e no metabolismo de neurotransmissores, como a noradrenalina, a dopamina, a serotonina e a acetilcolina (Stahl, 2001). Os estrógenos também agiriam sobre neuropeptídeos (por exemplo, fator de liberação de corticotrofinas [CRF], neuropeptídeo Y [NPY]), influenciando também a modulação de outras atividades, como a termorregulação em centros hipotalâmicos, e o controle da saciedade, do apetite e da pressão arterial sanguiínea. Assim, em períodos de variações abruptas dos níveis de estrógenos circulantes, ocorreria maior vulnerabilidade para o desenvolvimento de transtornos psíquicos na mulher, particularmente de cognição e do humor.

Em relação à utilização de doses suprafisiológicas de estrogênios, são necessários mais estudos que comprovem sua eficácia em mulheres que estão amamentando. Essa modalidade de tratamento não é a primeira escolha ao se considerarem riscos e benefícios do tratamento estrogênico para mulheres em fase reprodutiva.

\section{Psicoterapia}

Muitas mulheres abandonam ou não aceitam o tratamento farmacológico quando descobrem estar grávidas, e conforme a história de evolução e menor gravidade da doença, a psicoterapia pode ser uma boa opção de tratamento.

A psicoterapia também pode ser uma aliada no que diz respeito a medidas de descontinuação ou redução de dose no tratamento farmacológico, diminuindo o risco de recaídas ou os sintomas depressivos na gestação. No entanto, é importante ressaltar que não é adequado descontinuar a farmacoterapia em casos mais graves ou recorrentes. Episódios depressivos leves ou depressão menor podem ter boa resposta ao tratamento psicoterápico, sendo benéfico às pacientes e ao feto tentar inicialmente o tratamento não farmacológico nesses casos.

Newport et al. (2002) realizaram um estudo com 576 mulheres no pós-parto. Foram estudadas as abordagens psicoterápicas mais significativas na redução da sintomatologia nesses casos e verificaram-se duas modalidades mais efetivas: a psicoterapia interpessoal, que foca o seu trabalho nos sintomas depressivos da 
paciente e no seu relacionamento com o mundo, assim como o rompimento da barreira interpessoal; e a terapia cognitivo-comportamental (TCC), que tem como objetivo ajudar o paciente a solucionar seus comportamentos e cognições disfuncionais por meio da aprendizagem e da reestruturação cognitiva.

A TCC tem como base avaliar quais são as idéias, os pensamentos e as emoções que a pessoa possui sobre si mesma e que se encontram distorcidos, provocando uma cadeia de reações comportamentais disfuncionais.

Em pacientes deprimidos, é comum aparecerem relatos de idéias, pensamentos e sentimentos que acabam deixando o paciente com humor alterado, refletindo-se assim diretamente na vida dele e dos que o rodeiam. Esses pensamentos denominados automáticos são o cerne da teoria cognitivo-comportamental. A mudança desses pensamentos, muitas vezes distorcidos da realidade, faz com que o paciente com depressão passe a reavaliar sua condição e reestruturar seu cotidiano.

No caso das mulheres que apresentam depressão pós-parto, é extremamente comum apresentarem pensamentos e sentimentos relacionados a questões referentes aos cuidados do bebê e à sua situação atual. Dessa maneira, a atuação da TCC visa avaliar e reestruturar tais cognições que refletem em seu comportamento.

Meager (1996) detectou melhora significativa em gestantes e puérperas que foram submetidas à TCC em grupos de terapia, baseando-se nas escalas de Edinburgh, no Inventário de Beck para Depressão e no Profile of Mood States.

Spinelli e Endicott (2003) realizaram um estudo com gestantes, comparando dois grupos diferentes: 0 primeiro foi submetido a técnicas de educação familiar e o segundo, à psicoterapia interpessoal. As mulheres do segundo grupo foram submetidas a sessões semanais de 45 minutos, durante 16 semanas. Obtiveram-se os seguintes resultados: baseando-se na escala de Edinburgh, notou-se melhora em $11,8 \%$ das pacientes submetidas a técnicas de educação familiar e em 33,3\% das mulheres submetidas à psicoterapia interpessoal. Em relação à escala de Hamilton, notou-se melhora em $29,4 \%$ no primeiro e $52,4 \%$ no segundo grupo, respectivamente. Tendo-se como base o Inventário de Depressão de Beck, houve melhora em $23,5 \%$ das pacientes do primeiro grupo, enquanto $52,4 \%$ das pacientes submetidas à psicoterapia interpessoal melhoraram.

\section{Eletroconvulsoterapia (ECT)}

Gestantes severamente deprimidas, com idéias suicidas, quadros de mania, catatonia ou psicose podem necessitar de internação e, com freqüência, o tratamento de escolha nesses casos é a ECT. Duas revisões recentes constataram que seu uso é seguro e eficaz durante a gestação. Em uma revisão com 300 casos tratados com ECT durante a gestação, publicados nos últimos 50 anos, houve relato de quatro trabalhos de parto prematuros apenas. Não houve nenhum caso de ruptura prematura de placenta (Nonacs e Cohen, 2002).

Pesquisas recentes sugerem que o risco é mínimo durante a gravidez, tanto em relação à própria ECT quanto aos medicamentos utilizados durante o procedimento, fazendo da ECT um recurso útil no tratamento de transtornos psiquiátricos em gestantes. Maletzky (2004) realizou um estudo de revisão de 27 casos, descrevendo que, em 4 destes, a condição de gestante foi a principal indicação para a escolha de ECT como medida terapêutica, escolha esta realizada em função da segurança do método em relação aos riscos que podem haver quando ocorrem transtornos depressivos nessa fase. A ECT seria um procedimento adequado também como alternativa à farmacoterapia convencional às mulheres que não poderiam ser expostas a determinados tipos de medicações durante a gravidez, ou àquelas que não responderam ao tratamento farmacológico.

\section{Conclusão}

Os transtornos psiquiátricos na gestação e no puerpério são mais comuns do que se imagina, e muitos casos ainda são subdiagnosticados. Tem-se dado importância crescente ao tema, e pesquisas recentes têm focado também o prejuízo que essas patologias podem ocasionar não só à saúde da mãe, mas também ao desenvolvimento do feto, ao trabalho de parto e à saúde do bebê. Múltiplos fatores de risco estão envolvidos, mas a etiologia exata ainda não foi estabelecida. Esses transtornos costumam acometer pacientes que já tenham história de patologia psiquiátrica prévia, portanto, uma boa medida de prevenção é o tratamento adequado desses episódios. As medidas de tratamento ainda são amplamente discutidas, devendo-se levar em consideração a relação risco-benefício, sendo, assim, o bom senso do médico um aliado importante quanto à escolha do tratamento nesses casos. 
AREIAS, M.E. et al. - Comparative incidence of depression in women and men, during pregnancy and after childbirth. Validation of the Edinburgh Postnatal Depression Scale in Portuguese mothers. Br J Psychiatry 169 (1): 30-35, 1996.

Austin, M.P. et al. - Antenatal screening for the prediction of postnatal depression: validation of a psychosocial Pregnancy Risk Questionnaire. Acta Psychiatr Scand 112 (4): 310-317, 2005.

BazIRE, S. - Psychotropic drug directory. Reino Unido: Quay Books, 2002.

BЕСK, C.T. - The lived experience of postpartum depression: a phenomenological study. Nurs Res 41 (3): 166-170, 1992.

BECK, C.T. - Postpartum depressed mothers' experiences interacting with their children. Nurs Res 45 (2): 98-104, 1996.

BECK, C.T. - A checklist to identify women at risk for developing postpartum depression. J Obstet Gynecol Neonatal Nurs 27 (1): 39-46, 1998.

Beck, C.T.; Gable, R.K. - Postpartum Depression Screening Scale: development and psychometric testing. Nurs Res 49 (5): 272-282, 2000.

BeCK, C.T.; Gable, R.K. - Comparative analysis of the performance of the Postpartum Depression Screening Scale with two other depression instruments. Nurs Res 50 (4): 242-250, 2001.

Bennett, H.A. et al. - Prevalence of depression during pregnancy: systematic review. Obstet Gynecol 103 (4): 698-709, 2004.

BLoch, M.; DALY, R.C.; RuBinow, D.R. - Endocrine factors in the etiology of postpartum depression. Compr Psychiatry 44 (3): 234-246, 2003.

Bцосн, M. et al. - Risk factors associated with the development of postpartum mood disorders. J Affect Disord 88 (1): 9-18, 2005.

Botega, N.J. et al. - Prática psiquiátrica no hospital geral: interconsulta e emergência. 2.ed. Porto Alegre: Artmed, pp. 341-354, 2006.

Boyce, P.; Hickey, A. - Psychosocial risk factors to major depression after childbirth. Soc Psychiatry Psychiatr Epidemiol 40 (8): 605-612, 2005. Disponível em: www. springerlink.com.

BuIst, A.E. et al. - To screen or not to screen - That is the question in perinatal depression. Med J Aust 177 (suppl): 101-105, 2002.

CAntilino, A. - Tradução para o português e estudo de validação da Postpartum Depression Screening Scale na população brasileira. Dissertação (Mestrado). Universidade Federal de Pernambuco, Recife, 2003.
Cantilino, A. et al. - Validação da Escala de Depressão Pósparto de Edinburgh (versão em postuguês) no Nordeste do Brasil. In: Resumos do XXI Congresso Brasileiro de Psiquiatria. XXI Congresso Brasileiro de Psiquiatria, 2003, Goiânia.

Cantilino, A. et al. - Translation, validation and cultural aspects of Postpartum Depression Screening Scale in Brazilian Portuguese. Transcult Psychiatry (no prelo).

Chaudron, L.H; Pies, R.W. - The relationship between postpartum psychosis and bipolar disorder: a review. J Clin Psychiatry 64 (11): 1284-1292, 2003.

CLARK, G. - Discussing emotional health in pregnancy: the Edinburgh Postnatal Depression Scale. Br J Community Nurs 5 (2): 91-98, 2000.

Commitee on Drugs of American Academy of Pediatrics. - Use of psychoactive medication during pregnancy and possible effects on the fetus and newborn. Pediatrics 105 (4 Pt. 1): 880-887, 2000.

Cox, J.L.; Holden, J.M.; SAgovsky, R. - Detection of postnatal depression: Development of the 10-item Edinburgh Postnatal Depression Scale. Br J Psychiatry 150: 782-786, 1987.

Eletroconvulsoterapia. In: PsiqWeb. Disponível em: www. psiqweb.med.br. Revisto em 2005.

EpPerson, C.N. - Postpartum major depression: detection and treatment. Am Fam Physician 59 (8): 2247-2254, 2259-2260, 1999.

Fergerson, S.S.; Jamieson, D.J.; Lindsay, M. - Diagnosing postpartum depression: can we do better? Am J Obstet Gynecol 186 (5): 899-902, 2002.

GodFroId, I.O. et al. - Sleep during postpartum depression. Encephale 23 (4): 262-266, 1997.

GoLD, L.H. - Postpartum disorders in primary care: diagnosis and treatment. Prim Care 29 (1): 27-41, 2002.

HaberG, M., Matheson, I. - Antidepressive agents and breast feeding. Tidsskr Nor Laegeforen 117 (27): 3952-3955, 1997.

Hendrick, V. et al. - Postpartum and nonpostpartum depression: differences in presentation and response to pharmacologic treatment. Depress Anxiety 11 (2): 66-72, 2000.

HoLcomb JR, W.L. et al. - Screening for depression in pregnancy: characteristics of the Beck Depression Inventory. Obstet Gynecol 88 (6): 1021-1025, 1996.

JABLENSKY, A.V. et al. - Pregnancy, delivery, and neonatal complication in a population cohort of women with schizophrenia and major affective disorders. Am J Psychiatry 162 (1): 79-91, 2005. 
JenNings, K.D. et al. - Thoughts of harming infants in depressed and nondepressed mothers. J Affect Disord 54 (1-2): 21-28, 1999.

Josefsson, A. et al. - Prevalence of depressive symptoms in late pregnancy and postpartum. Acta Obstet Gynecol Scand 80 (3): 251-255, 2001.

LABAD, J. et al. - Female reproductive cycle and obsessivecompulsive disorder. J Clin Psychiatry 66 (4): 428-435, quiz 546, 2005.

Maletzky, B.M. - Related articles, links. The first-line use of eletroconvulsive therapy in major affective disorders. $J$ ECT 20 (2): 112-117, 2004.

MC EwEN, B.S- Related Articles, Links. Clinical review 108: The molecular and neuroanatomical basis for estrogen effects in the central nervous system. J Clin Endocrinol Metab 84(6): 1790-7, 1999.

Meager, I.; Milgrom, J. - Group treatment for postpartum depression: a pilot study. Aust N Z J Psychiatry 30 (6): 852-860, 1996.

MurRaY, D. - Related articles, links - Estrogen and postnatal depression. Lancet 347 (9006): 918-919, 1996.

NEWPORT, D.J. et al. - The treatment of postpartum depression: minimizing infant exposures. J Clin Psychiatry 63 (suppl. 7): 31-44, 2002.

Nonacs, R.; Cohen, L.S. - Postpartum mood disorders: diagnosis and treatment guidelines. J Clin Psychiatry 59 (suppl. 2): 34-40, 1998.

Nonacs, R.; Cohen, L.S. - Postpartum psychiatric syndromes. In: Sadock, B.J.; Sadock, V.A. (eds.) Kaplan \& Sadock's Comprehensive Textbook of Psychiatry. 7.ed. Filadélfia: Lippincott Williams \& Wilkins, pp. 1276-1283, 2000.

Nonacs, R; Cohen, L.S. - Depression during pregnancy: diagnosis and treatment options. J Clin Psychiatry 63 (suppl. 7): 24-30, 2002.

Northcott, C.J.; Stein, M.B. - Panic disorder in pregnancy. $J$ Clin Psychiatry 55 (12): 539-542, 1994.

O'HarA, M.W.; Swaln, A.M. - Rates and risk of postpartum depression - A meta-analysis. Int Rev Psych 8: 37-54, 1996.

Organização Mundial da Saúde (org.). Classificação de Transtornos Mentais e de Comportamento da CID-10: Descrições Clínicas e Diretrizes Diagnósticas. Tradução de Dorgival Caetano. Porto Alegre: Artes Médicas, p. 191, 1993.

Righetti-Veltema, M. et al. - Postpartum depression and mother-infant relationship at 3 months old. J Affect Disord 70 (3): 291-306, 2002.
Ross, L.E. et al. - Measurement issues in postpartum depression part 1: anxiety as a feature of postpartum depression. Arch Women Ment Health 6 (1): 51-57, 2003.

Ryan, D.; MıLIs, L.; MısRı, N. - Depression during pregnancy. Canadian Family Physician/Le Médecin de famille canadien 51: 1087-1093, 2005.

Santos, M.F.; Martins, F.C.; Pasqualı, L. - Escala de autoregistro de depressão pós-parto: estudo no Brasil. In: Gorenstein, C.; Andrade, L.H.; Zuardi, A.W. (eds.). Escalas de Avaliação Clínica em Psiquiatria e Psicofarmacologia. São Paulo: Lemos, pp. 97-101, 2000.

SENG, J.S. et al. - Posttraumatic stress disorder and pregnancy complications. Obstet Gynecol 97 (1): 17-22, 2001.

Schmiege, S.; Russo, N.F. - Depression and unwanted first pregnancy: longitudinal cohort study. BMJ 331 (7528): 1303, 2005.

SholomsKAS, D.E. et al. - Postpartum onset of panic disorder: a coincidental event? J Clin Psychiatry 54 (12): 476-480, 1993.

SpINELLI, M.G. - Maternal infanticide associated with mental illness: prevention and the promise of saved lives. $A m \mathrm{~J}$ Psychiatry 161 (9): 1548-1557, 2004.

SpineluI, M.G.; EndicotT, J. - Controlled clinical trial of interpersonal psychotherapy versus parenting education program for depressed pregnant women. Am J Psychiatry 160 (3): 555-562, 2003.

StAHL, S.M- Related Articles, Links. Natural estrogen as an antidepressant for women. J Clin Psychiatry62(6): 404-5, 2001. PMID: 11465515 (Pub Med-indexed for Medline).

Szigethy, E.M.; RuIz, P. - Depression among pregnant adolescents: an integrated treatment approach. Am J Psychiatry 158 (1): 22-27, 2001.

UgARIZA, D.N. - Screening for postpartum depression. $J$ Psychosoc Nurs Ment Health Serv 38 (12): 44-51, 2000.

Williams, K.E.; Koran, L.M. - Obsessive-compulsive disorder in pregnancy, the puerperium, and the premenstruum. $J$ Clin Psychiatry 58 (7): 330-334, 1997.

Williams, M.; WoOltorton, E. - Paroxetine (Paxil) and congenital malformations. CMAJ 173 (11): 1320-1321, 2005.

WISNER, K.L. et al. - Obsessions and compulsions in women with postpartum depression. J Clin Psychiatry 60 (3): 176-180, 1999.

WISNER, K.L. et al. - Risk-benefit decision making or treatment of depression during pregnancy. Am J Psychiatry 157: 1933-1940, 2000. 